\title{
NONLINEAR EVOLUTION OF WAVE PACKETS IN MAGNETIC FLUIDS
}

\author{
BY \\ S. K. MALIK * AND M. SINGH \\ Simon Fraser University, Burnaby, British Columbia, Canada
}

\begin{abstract}
The propagation of wave packets on the surface of a magnetic fluid of finite depth is considered in $(2+1)$ dimensions. It is shown that the evolution of the envelope is governed by two coupled partial differential equations with cubic nonlinearity. The stability analysis reveals the existence of more than one region of instability. The enveloping soliton and the "waveguide" solutions are derived in the regions of instability wherever they exist in one space dimension. The instability regions are sensitive to the applied magnetic field strength. The evolution of the envelope is governed by a $(2+1)$-dimensional nonlinear equation which leads to a self-focussing singularity. Examined also is the long wave/short wave resonant interaction in magnetic fluids. Our study shows that the application of the magnetic field decreases the region of instability where this resonance occurs.
\end{abstract}

1. Introduction. Because of the wide range of important industrial applications, there has been a growing interest in recent years in the study of the magnetic fluids when subjected to the normal and the tangential magnetic fields. The theoretical as well as the experimental development of the linear theory governing the effect of a tangential magnetic field on the interface between magnetic fluids was initially conducted by Zelazo and Melcher [1] (see also [2-4]), where it was shown that the magnetic field has a stabilizing influence. Later, Malik and Singh [5, 6], investigated the nonlinear evolution of the wave packets in one-dimensional space on the free surface of a magnetic fluid. It was proved that there exist different regions of instability and that the magnetic field and permeability have varying effects in these regions of instability. Using a geometrical method, Shen and Sun [7] examined the surface waves for a ferromagnetic fluid of variable depth, and reported the existence of edge waves over a uniformly sloping bottom.

The aim of this paper is to present the nonlinear wave propagation on the free surface of a magnetic fluid of finite depth in $(2+1)$ dimensions when it is subjected to a tangentially applied magnetic field. In hydrodynamics, such a problem has already been studied by Davey and Stewartson [8], Djordjevic and Redekopp [9], Ablowitz and Segur [10].

Received May 28, 1988.

* Permanent address: Department of Mathematics. Panjab University, Chandigarh. India.

(C) 1990 Brown University 
In Sec. 1, we outline the fundamental equations and the perturbation procedure for obtaining the successive higher-order nonlinear equations with the use of the method of multiple scales. The two coupled equations which yield the evolution of the amplitude are derived in Sec. 2. These equations, in general, are not integrable. However, these equations can be integrated by the inverse scattering technique [11] in the long wave limit.

The stability of the two coupled equations for the evolution of the amplitude is put forth in Sec. 3. In Sec. 4, we discuss the stability of the solution. In confining to one space dimension and for moderate values of the magnetic field strength, there exist three regions of instability and three regions of stability. If the propagation is along the direction of the applied magnetic field, the envelope solitons are shown to exist in unstable regions. With the increase in either the strength of the applied magnetic fluid or the permeability of the magnetic fluid, the height of the solitons increases in some regions and decreases in others. However, the speed of the solitons in all regions always increases. On the other hand, if the wave packet is modulating in the $y$-direction only, the 'waveguide' solutions, similar to nonlinear Fraunhofer diffraction in Optics [12], are generated which are in fact envelope solitons. The height and speed of the "waveguide" both increase with the increase in the magnetic field strength.

The one-dimensional solutions mentioned above are unstable against transverse perturbations. The growth rate of such an instability is influenced by the applied magnetic field, and may give rise to a self-focussing phenomenon [13]. (See also [1416].) The wave field becomes singular at a certain point, referred to as the "focus." At this singularity, the wave amplitude becomes infinite and may lead to the appearance of turbulent bursts. This phenomenon has been developed in Sec. 5 .

In Sec. 6, we have investigated the interaction of the long and the short wave resonance in magnetic fluids. Benney $[17,18]$, has discussed this type of interactions for ideal fluids where the physical mechanism is that of three waves interaction out of which two are short capillary waves and the third being a long gravity wave. This process is operative when the phase velocity of the long gravity wave coincides with the group velocity of the short waves, and the energy transfer takes place from one mode to another. Benney [18] describes these interactions by two coupled evolution equations, and obtains a permanent wave solution with the capillary gravity wave phase-locked to the crest of the gravity wave. The short waves act as a generator of long waves by means of the sideband instability process analogous to that of Benjamin and Feir [19]. Another interesting model dwelling on wave interactions has been discussed by Newell [20] which admits solution through inverse scattering transform. Djordjevic and Redekopp [9] have also studied the resonance mechanism in the shallow water limit. In our study of extending the above phenomena for magnetic fluids under the influence of an applied magnetic field, the conditions for triad wave interactions are also met. Like [9], we also employ the time scales for interactions which are faster than those used by Benney [17]. We show that the presence of the magnetic field enhances the region of stability. The long waves which, if they are initially absent, can be suppressed much more easily in magnetic fluids by either 
increasing the strength of the applied magnetic field or using the magnetic fluids with higher permeabilities.

2. Fundamental equations. We consider an inviscid, homogeneous, and incompressible magnetic fluid of uniform depth $b$. The magnetic fluid with density $\rho_{1}$ and permeability $\mu_{1}$ occupies the half space $z<0$, whereas the medium $z>0$ has negligible density and magnetic permeability $\mu_{2}$. The system is subjected to an applied uniform magnetic field $\mathbf{H}(1,0,0)$ along the fluid interface, and the gravitational force $\mathbf{g}(0,0,-1)$. The magnetic fluid is assumed to be initially quiescent with linear magnetization properties.

We shall investigate the propagation of weakly nonlinear waves, confining ourselves to a wave train that has a principal direction along the $x$-axis although modulations in both the $x$ and $y$ directions shall be allowed.

The basic equation governing the irrotational motion is

$$
\nabla^{2} \phi=0 \text { for }-\infty<x<\infty,-\infty<y<\infty, \quad \text { and }-b<z<\eta(x, y, t) .
$$

Here, $\phi(x, y, z, t)$ is the velocity potential $(\mathbf{v}=\nabla \phi)$, and $z=\eta(x, y, t)$ is the elevation of the free surface. The perturbation generates an additional irrotational magnetic field $\mathbf{h}$ represented by the potential $\psi(x, y, z, t)(\mathbf{h}=-\nabla \psi)$ such that

$$
\nabla^{2} \psi^{(1)}=0, \quad-b<z<\eta(x, y, t)
$$

and

$$
\nabla^{2} \psi^{(2)}=0, \quad \eta(x, y, t)<z<\infty .
$$

Since the motion away from the interface and at $z=-b$ vanishes, we have

$$
\begin{gathered}
|\nabla \phi| \rightarrow 0, \quad\left|\nabla \psi^{(1)}\right| \rightarrow 0 \quad \text { as } z \rightarrow-b, \\
\left|\nabla \psi^{(2)}\right| \rightarrow 0 \quad \text { as } z \rightarrow \infty .
\end{gathered}
$$

At the free surface $z=\eta(x, y, t)$ the relevant boundary conditions for this threedimensional problem are

$$
\begin{gathered}
\frac{\partial \eta}{\partial t}-\frac{\partial \phi}{\partial z}+\frac{\partial \phi}{\partial x} \frac{\partial \eta}{\partial x}+\frac{\partial \phi}{\partial y} \frac{\partial \eta}{\partial y}=0 \\
\mu H_{n}^{(1)}=H_{n}^{(2)} \\
H_{T}^{(1)}=H_{T}^{(2)}
\end{gathered}
$$

and

$$
\begin{aligned}
\frac{\partial \phi}{\partial t}+ & g \eta+\frac{1}{2}(\nabla \phi)^{2} \\
= & \frac{T}{\rho}\left[\frac{\partial^{2} \eta}{\partial x^{2}}\left(1+\left(\frac{\partial \eta}{\partial y}\right)^{2}\right)+\frac{\partial^{2} \eta}{\partial y^{2}}\left(1+\left(\frac{\partial \eta}{\partial x}\right)^{2}\right)-2 \frac{\partial^{2} \eta}{\partial x \partial y} \frac{\partial \eta}{\partial x} \frac{\partial \eta}{\partial y}\right] \\
& \times\left(1+\left(\frac{\partial \eta}{\partial x}\right)^{2}+\left(\frac{\partial \eta}{\partial y}\right)^{2}\right)^{-3 / 2} \\
& +\frac{\mu_{2}(\mu-1)}{8 \pi \rho}\left[\left(H^{(1)}\right)^{2}+(\mu-1)\left(H_{n}^{(1)}\right)^{2}\right] .
\end{aligned}
$$


Here, $\mu=\mu_{1} / \mu_{2}$, and $T$ is the surface tension whereas $H^{(1)}$ and $H^{(2)}$ are the magnetic fields in two media. In the derivation of the last term in Eq. (9), we have eliminated the magnetic field term $H^{(2)}$ by using Eqs. (7)-(8). The quantities $H_{n}$ and $H_{T}$ represent the normal and the tangential components of the magnetic field, respectively.

To obtain approximate solutions governed by Eqs. (1)-(9) for small but finite amplitudes, we use the method of multiple scales and therefore introduce

$$
\begin{gathered}
x=\varepsilon^{n} x_{n}, \quad y=\varepsilon^{n} y_{n+1}, \quad t=\varepsilon^{n} t_{n}, \quad n=0,1,2, \\
\Phi(x, y, z, t)=\sum_{n=1}^{N} \varepsilon^{n} \phi_{n}\left(z, x_{0}, x_{1}, x_{2}, y_{1}, y_{2} ; t_{0}, t_{1}, t_{2}\right) \\
+O\left(\varepsilon^{N+1}\right),
\end{gathered}
$$

$\Phi$ can be any of the physical quantities $\phi, \psi, \eta$, and where the small parameter $\varepsilon$ is the steepness ratio of the wave. We shall also assume that $(k b)^{2} \gg \varepsilon$, where $k$ is the characteristic wavenumber along the $x$ direction.

3. Derivation of the amplitude equation. The solution to the first-order or linear problem, as developed in (6), is

$$
\begin{gathered}
\phi_{1}=-i \omega k^{-1} \cosh k(z+b) F+c c+B^{(1)}, \\
\psi_{1}^{(1)}=i B H \sinh k(z+b) F+c c \\
\psi_{1}^{(2)}=i B H \exp (-k z) A \exp (i \theta)+c c, \\
\eta_{1}=A \exp (i \theta)+c c
\end{gathered}
$$

where

$$
\begin{gathered}
F=(\sinh (k b))^{-1} A \exp (i \theta), \\
\theta=k x_{0}-\omega t_{0}, \\
B=\frac{(1-\mu)}{\mu \sigma(b)+1},
\end{gathered}
$$

with

$$
\sigma(b)=\operatorname{coth}(k b)
$$

The amplitude $A$ is a function of $x_{1}, x_{2}, y_{1}, y_{2}, t_{1}$, and $t_{2}$. The constant $B^{(1)}$, which is a function of the slower scales, has been introduced to eliminate the secularities at the higher orders. The solution above gives rise to the dispersion relation

$$
D(\omega, k)=\frac{-\omega^{2}}{k} \sigma(b)+\frac{T}{\rho} k^{2}+g+\frac{(\mu-1)^{2} k V_{4}^{2}}{\mu \sigma(b)+1}=0,
$$

where

$$
V_{1}^{2}=\frac{\mu_{2} H^{2}}{4 \pi \rho}
$$


For higher-order problems, we again follow the procedure outlined by Malik and Singh [6]. The requirement that the second-order solution be bounded yields the nonsecularity condition

$$
\frac{\partial A}{\partial t_{1}}+V_{g} \frac{\partial A}{\partial x_{1}}=0
$$

where $V_{g}=\frac{d w}{d k}$ is the group velocity of the wave packet. The quantity $B^{(1)}$, which represents the induced mean motion or the zero frequency correction to slow modulation of the fundamental mode, is given in the second-order problem by

$$
\frac{\partial^{2} B^{(1)}}{\partial t_{1}^{2}}-g b\left(\frac{\partial^{2} B^{(1)}}{\partial x_{1}^{2}}+\frac{\partial^{2} B^{(1)}}{\partial y_{1}^{2}}\right)=2\left(V_{g} q_{1}+\omega g \sigma(b)\right) \frac{\partial|A|^{2}}{\partial x_{1}},
$$

where

$$
q_{1}=\frac{1}{2}\left[\omega^{2}\left(\sigma^{2}(b)-1\right)+(\mu-1) k^{2} V_{A}^{2}\left\{\left(\mu \sigma^{2}(b)+1\right) B^{2}+(\mu-1)+2 B \mu \sigma(b)\right\}\right] .
$$

If we put $V_{A}=0$ in Eq. (23), we recover the result of Davey and Stewartson [8] for hydrodynamics.

Introducing the transformations $\xi=\left(x_{1}-V_{g} t_{1}\right)$ and $\eta=y_{1}$, Eq. (23) reduces to

$$
\left(g b-V_{g}^{2}\right) \frac{\partial^{2} B^{(1)}}{\partial \xi^{2}}+g b \frac{\partial^{2} B^{(1)}}{\partial \eta^{2}}=-2\left(q_{1} V_{g}+\omega g \sigma(b)\right) \frac{\partial|A|^{2}}{\partial \xi} .
$$

Equation (25) is elliptic for $M\left(=V_{g}^{2} / g b\right)<1$ and hyperbolic for $M>1$. For water waves, the flow is subsonic when $M<1$. However, in ferrofluids even for $T=0$, the flow is supersonic $(M>1)$ for moderately strong values of the magnetic field. The surface tension effect is required to investigate the significant interactions of large and small waves. It is shown in Fig. 1 that the transition curve $S(k)=V_{g}^{2}-g b=0$ changes significantly due to magnetization for small values of $k b$. Unlike negative dispersion obtained for water waves, we get here a positive dispersion for small values of $k b$, leading to lump solutions which are localized waves in $(2+1)$ dimensions [26].

To develop the amplitude modulation for the progressive waves, we need to go to the third-order problem. By substituting the first- and second-order solutions into that of the third order, we obtain the solvability condition

$$
i \frac{\partial A}{\partial \tau}+P \frac{\partial^{2} A}{\partial \xi^{2}}+P_{1} \frac{\partial^{2} A}{\partial \eta^{2}}=Q_{1}|A|^{2} A+Q_{2} A \frac{\partial B^{(1)}}{\partial \xi},
$$

where

$$
\begin{gathered}
P=\frac{1}{2} \frac{\partial V_{g}}{\partial k}, \quad t_{2}=\tau, \\
P_{1}=\frac{V_{g}}{2 k} \geq 0,
\end{gathered}
$$



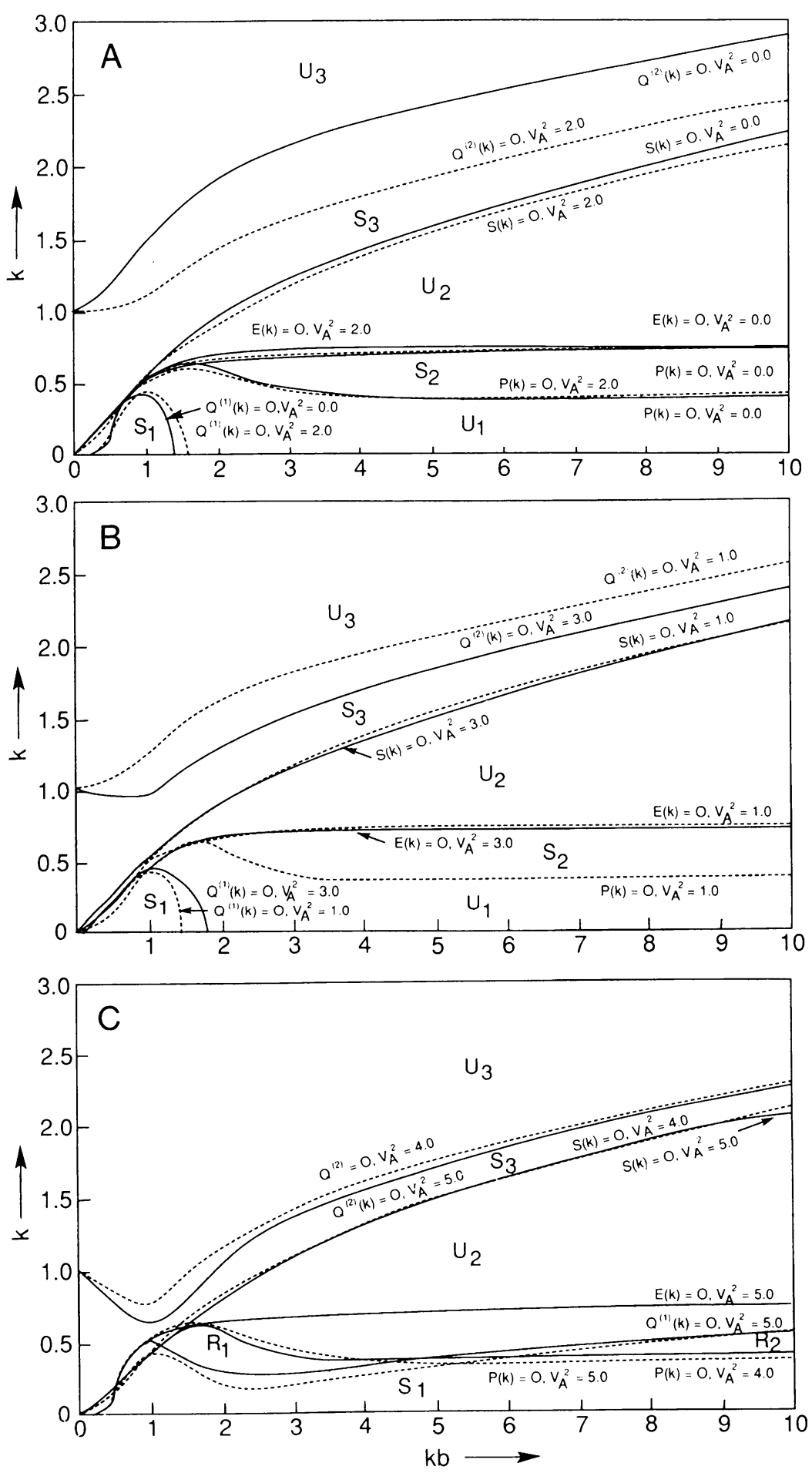

FIG. 1. The stability diagram showing different regions of stability $S_{1}, S_{2}, S_{3}$ and regions of instability $U_{1}, U_{2}, U_{3}, R_{1}$, and $R_{2}$ for the values of $I_{A}^{2}=0,1,2,3,4,5$ with $\mu=1.5$. 


$$
\begin{array}{r}
Q_{1}=\frac{-k}{2 \omega \sigma(b)}\left[\omega^{2}\left(2(\Lambda-k \sigma(b))(1-\sigma(b) \sigma(2 b))+\Lambda+\xi_{2}-\frac{5}{2} k \sigma(b)\right)+\frac{3}{2} k^{4} T\right. \\
-\omega^{2} \sigma(b)\left(\Lambda(2 \sigma(2 b)+\sigma(b))+\xi_{2} \sigma(b)+\frac{k}{2}(3-4 \sigma(b) \sigma(2 b))\right) \\
+\frac{k^{2} V_{A}^{2}}{2}(\mu-1)\left[\begin{array}{l}
4 q_{2}((\mu \sigma(b)(\sigma(2 b)+1) B+(\mu+1) \sigma(2 b)) \\
+2 B \Lambda(2 \mu-1) \sigma(b)-2(1+\mu)+2 B \xi_{2} \sigma(b) \\
+k B \sigma(b)(4 B(\mu+1)+3) \\
\left.\left.+4 k(\mu-1) B+\frac{2\left(l_{1}-l_{2}\right) \sigma(b)}{\mu \sigma(b)+1}\right]\right]
\end{array}\right.
\end{array}
$$

with

$$
\begin{gathered}
l_{1}=\left(\Lambda+\xi_{2}\right)(\mu-1)^{2} B_{1}+2\left(q_{3}-\mu q_{2}\right)-\frac{3}{2} k B_{1}(1-\mu), \\
l_{2}=2\left(q_{2} \sigma(2 b)+q_{3}\right)+\left(\xi_{2}-\lambda\right) B(1+\sigma(b)), \\
q_{2}=\frac{(1-\mu)\left[\Lambda-k(\mu+\sigma(b)) B_{1}\right]}{\mu \sigma(2 b)+1}, \\
q_{3}=q_{2}+k B_{1}(\sigma(b)+1)(1-\mu), \\
\xi_{2}=-2 g^{-1} q_{1}, \quad B_{1}=(1+\mu \sigma(b))^{-1}, \\
\Lambda=\frac{1}{D(2 \omega, 2 k)}\left[\frac{\omega^{2}}{2}\left(3-\sigma^{2}(b)\right)-2 \omega^{2} \sigma(2 b) \sigma(b)\right. \\
+k^{2}(\mu-1) \frac{V_{A}^{2}}{2}\left\{-4 B(\mu+\sigma(b)) \times(\mu \sigma(2 b)+1)^{-1}\right. \\
\left.\left.\quad+B^{2}\left(1-\mu \sigma^{2}(b)\right)+(1-\mu)+2 B \sigma(b)(2-\mu)\right\}\right], \\
Q_{2}=-\frac{-2 \omega^{2}}{2 \omega \sigma(b)}\left\{\left[\omega^{2}\left(1-\sigma^{2}(b)\right)+\frac{k^{2} V_{A}^{2}}{2}(\mu-1)\left(2 B \sigma(b)+4 \frac{T k^{2}}{\rho}+\frac{2 k V_{A}^{2}(\mu-1)^{2}}{\mu \sigma(2 b)+1},\right.\right.\right. \\
\left.\left.+2 \frac{\left((\mu-1)^{2} B_{1}-B(1+\sigma(b)) \sigma(b)\right.}{\mu \sigma(b)+1}\right] V_{g} g^{-1}-2 \omega \sigma(b)\right\} .
\end{gathered}
$$

Equations (25) and (26) are the coupled nonlinear interaction equations. Since our interest is in waves propagating only in the principal $x$-direction, we have evaluated all the quantities at $l=0$, where $l$ is the wavenumber of waves propagating in the $y$-direction. By virtue of Eqs. (29) and (35), the nonlinear interaction coefficient $Q_{1}$ becomes singular whenever $D(2 \omega, 2 k)=0$. In magnetic fluids, this phenomenon can be studied further following the procedure outlined by Kant and Malik [21], except that shorter time scales than used in this paper shall be required. The interaction 
coefficient $Q_{2}$ also has a singularity when $M=1$. For this value of $M$, there is a resonance interaction between the long and the short waves. The solution in the vicinity of such a resonance phenomenon is obtained in Sec. 6 .

In this section, we consider $D(2 \omega, 2 k) \neq 0$ and $M \neq 1$.

Setting

$$
S=\left(2 V_{g} q_{1}+\omega \sigma(b)|A|^{2}\right)\left(g b-V_{g}^{2}\right)^{-1}+\frac{\partial B^{(1)}}{\partial \xi},
$$

and substitution for $S$ in Eqs. (25) and (26), yields

$$
\begin{gathered}
i \frac{\partial A}{\partial \tau}+P \frac{\partial^{2} A}{\partial \xi^{2}}+P_{1} \frac{\partial^{2} A}{\partial \eta^{2}}=Q|A|^{2} A+Q_{2} S A \\
\left(g b-V_{g}^{2}\right) \frac{\partial^{2} S}{\partial \xi^{2}}+g b \frac{\partial^{2} S}{\partial \eta^{2}}=g b\left(\frac{2 V_{g} q_{1}+\omega \sigma(b) g}{g b-V_{g}^{2}}\right) \frac{\partial^{2} A}{\partial \eta^{2}}
\end{gathered}
$$

where

$$
Q=Q_{1}+\frac{Q_{2}\left(2 V_{g} q_{1}+\omega \sigma(b) g\right)}{V_{g}^{2}-g b} .
$$

It is interesting to observe that $Q$ in Eq. (41) is singular at $V_{g}^{2}=g b$. Physically, it represents the resonance between the long and the short waves. Our solution is not valid in the vicinity of such a resonance. We aim to discuss this resonance in Sec. 6. For ideal fluids, such types of resonance have been investigated by Benney [18], Djordjevic and Redekopp [9], and Newell [20].

In deep magnetic fluids, i.e., $k b \rightarrow \infty$, Eqs. (25) and (26) furnish

$$
2 \frac{\partial A}{\partial \tau}+P_{1}^{\prime} \frac{\partial^{2} A}{\partial \xi^{2}}+P_{2}^{\prime} \frac{\partial^{2} A}{\partial \eta^{2}}=Q^{\prime}|A|^{2} A
$$

where

$$
\begin{aligned}
P_{1}^{\prime} & =\frac{1}{2} \frac{d V_{g}}{d k}, \\
P_{2}^{\prime} & =\frac{V_{g}}{2 k}, \\
Q^{\prime} & =\frac{k}{4 \omega}\left[2 \Lambda^{\prime}\left\{\omega^{2}-k^{2} V_{A}^{2} \frac{(\mu-1)}{(\mu+1)}+2 k\left(\omega^{2}-k^{2} V_{A}^{2}\right)\right\}-\frac{3}{2} T k^{4}\right] A^{2} \bar{A}, \\
\Lambda^{\prime} & =\left[-\omega^{2}+k^{2} V_{A}^{2}(\mu-1) /(\mu+1)\right]\left[2 k^{2} \frac{T}{\rho}-g\right]^{-1},
\end{aligned}
$$

along with the boundary condition that $A$ vanishes as $\xi^{2}+\eta^{2} \rightarrow \infty$. Equation (42) gives rise to a focussing singularity.

4. Stability of solution. We shall discuss now the solution of the coupled equations (25) and (26). With a view to describe the stability of the one-dimensional solution against transverse perturbations, we first review the solution in one space dimension only [6]. In the special case when the propagation is only in the $x$-direction and 

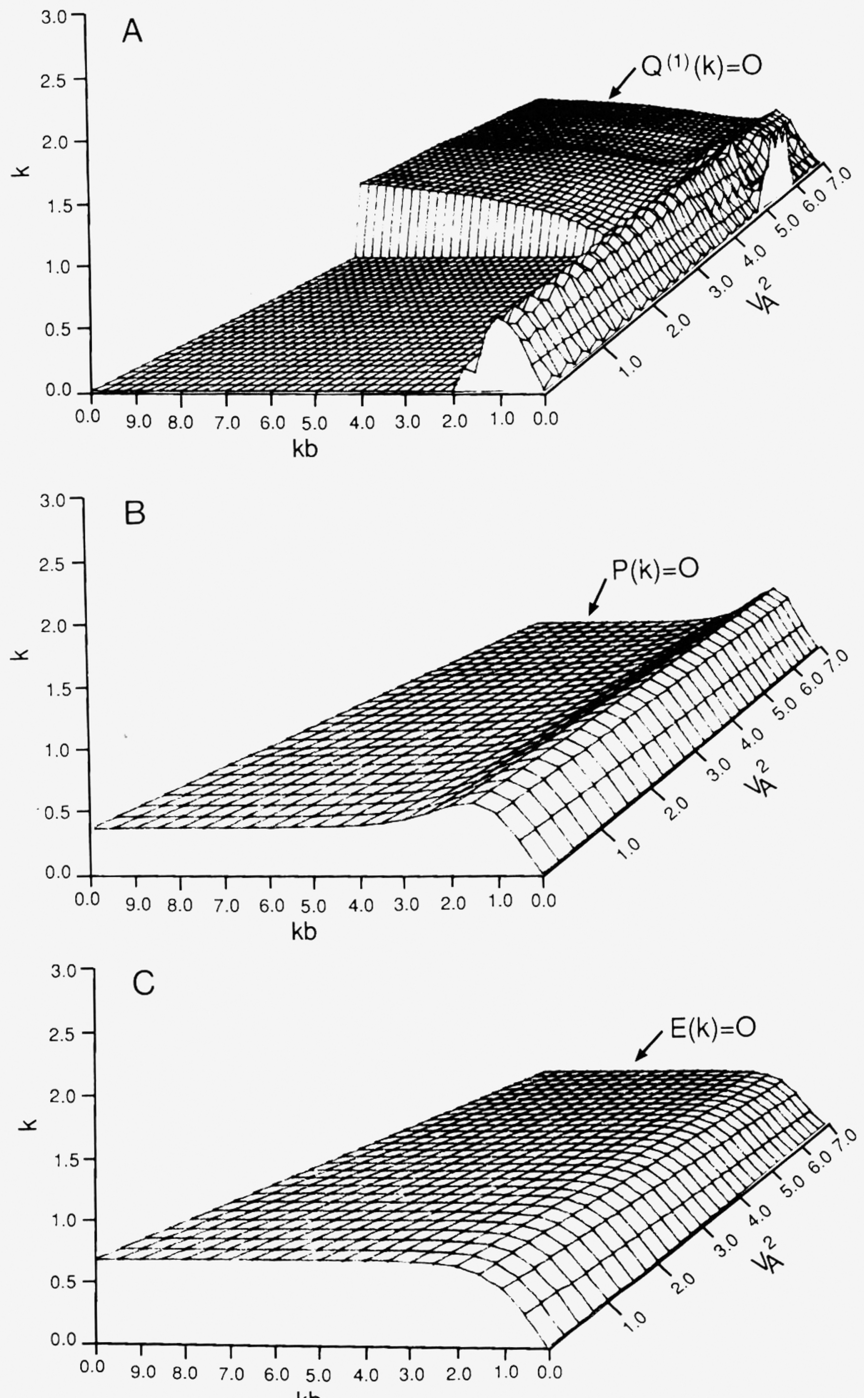

$\mathrm{kb}$

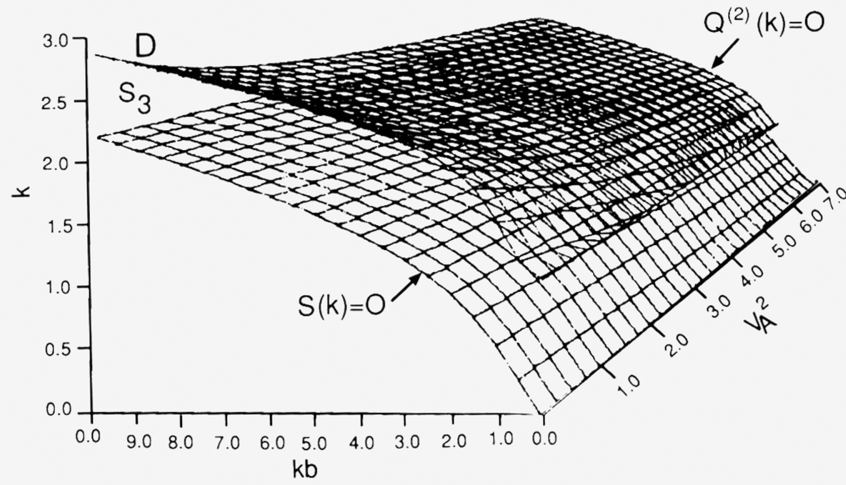

FIG. 2. The three-dimensional stability plots of various surfaces across which the coefficients $P, Q^{(1)}, E, S, Q^{(2)}$ change sign. 
when the modulation in the $y$-direction is not taken into account, Eq. (25) reduces to

$$
\frac{\partial B^{(1)}}{\partial \xi}=\frac{2\left(V_{g} q_{1}+\omega g \sigma(b)\right)}{\left(V_{g}^{2}-g b\right)}|A|^{2},
$$

and, therefore, with Eq. (26) we get

$$
i \frac{\partial A}{\partial \tau}+P \frac{\partial^{2} A}{\partial \xi^{2}}=Q|A|^{2} A .
$$

Malik and Singh [6] examined the stability of the solution (48) by normalizing all the physical quantities in Eq. (48) with respect to the characteristic length $(T / \rho g)^{1 / 2}$ and the characteristic time $\left(T / \rho g^{3}\right)^{1 / 2}$. Figure 1 shows the stability diagrams for $\mu=1.5$. The curves correspond to the group velocity $P$, the nonlinear interaction parameter $Q$, the second harmonic resonance $E(k)=D\left(2 \omega, 2 k, \mu, V_{A}^{2}\right)=0$, and resonance of the long and the short waves given by $S(k)=V_{g}^{2}-g b=0$ when the coefficients $P$ and $Q$ of Eq. (48) change sign across these curves. Here, it is interesting to note that there are two branches $Q^{(1)}(k)=0, Q^{(2)}(k)=0$ across which $Q(k)$ changes sign. The three-dimensional stability diagrams are exhibited in Fig. 2. Furthermore, the figures exhibit the existence of three regions of stability $S_{i}$ and three regions of instability $U_{i}, i=1,2,3$ for moderate strengths of the magnetic field. The higher values of the magnetic field generate two new regions of instability indicated as $R_{1}$ and $R_{2}$ in the figures.

In this paper, our objective is to investigate the stability of the soliton solutions of Eqs. (25) and (26) in $(2+1)$ dimensions. In one space dimensions, Eqs. (25) and (26) reduce to a single equation (48). The one soliton solution of Eq. (48) is given, as in [21], by

$$
A=a \operatorname{Sech}\left(\frac{a}{h}\left(\xi-2 b_{0} \tau\right)\right) \exp \left(i b_{0} \xi+i P\left(a^{2}-b_{0}^{2}\right) \tau\right),
$$

where $h=(2 P / Q)^{-1 / 2}$ represents the height of the soliton and where $a, b_{0}$ are constants. The constant $b$ is a measure of the correction to the basic wavenumber to the $O(\varepsilon)$, and is therefore taken equal to zero. The height of the soliton depends on the group velocity rate $P$ and the nonlinear interaction parameter $Q$. The height of the envelope soliton decreases in the region $U_{1}$ and increases in regions $U_{2}$ and $U_{3}$ when the magnetic field strength is increased. However, the speed of the envelope soliton increases with increase in the magnetic field strength in all the unstable regions. The number of these solitons and the structure of the associated tail is completely determined by the initial data. For a pulse initial condition, the tail is not very important and decays as $t^{-1 / 2}$ times the amplitude.

We now turn our attention to the "waveguide" solution of Eqs. (25) and (26) when the wave propagates in the principal direction of the $x$-axis and the modulation takes place in the $y$-direction. The "waveguide" solutions are essentially the envelope solitons. In such a case, $\partial^{2} A / \partial \xi^{2}=0$ and Eqs. (25) and (26) reduce to a onedimensional Schrödinger equation similar to (48) with $\xi$ replaced by $\eta$. Since $P_{1}>0$ and $Q_{1}<0$, these solutions exist in the regions $U_{2}, S_{3}$, and $U_{3}$. Both 
speed and height of these "waveguides" increase with increase in the magnetic field strength.

For a general case when $\partial^{2} A / \partial \xi^{2}$ and $\partial^{2} A / \partial \eta^{2}$ are both nonzero, there may be a crossover from one region to another.

We shall now discuss the stability of soliton solutions subject to transverse perturbations. Following procedures established by Zakharov and Rubenchik [23] and Ablowitz and Segur [10], we seek the solution of the form

$$
A=(\delta+u+i v) \exp \left(i P a^{2} \tau\right),
$$

where $u, v$ are assumed to be real and $\ll \delta$. With $A$ in Eq. (50), the Eqs. (25) and (26) are linearized. We further assume that

$$
u, v \propto \exp (i K \eta \pm i \Omega \tau) .
$$

The analysis of the stability of the solution can be simplified by looking for the solution in terms of odd and even functions of $\xi$, denoted respectively by the subscripts $(-)$ and $(+)$. In a medium with cubic nonlinearity, the growth rate for the even and the odd modes comes out to be

$$
\Omega_{+}^{2}=-2 P P_{1} a^{2}
$$

and

$$
\Omega_{-}^{2}=\frac{4}{3} P a^{2}\left[P_{1}+\frac{2 Q_{2}\left(V_{g} q_{1}+\omega g \sigma(b)\right)}{\left(g b-V_{g}^{2}\right)}\left|\frac{P}{Q}\right|\right] .
$$

For deep magnetic fluids, $Q_{2}=0$. We shall now investigate the effect of the increase in the magnetic field strength on the unstable regions $U_{i}, R_{1}$, and $R_{2}$ sketched in Fig. 1.

Firstly, we look at the regions $U_{1}, R_{1}$, and $R_{2}$. In the one-dimensional case, we have envelope solitons as $P Q<0$. This soliton which has amplitude $a$ is unstable against long perturbations which are antisymmetric in $\xi$. The growth rate of this perturbation with wavenumber $K$ is given by

$$
\Omega^{2}=-\frac{4}{3} K^{2} a^{2}|P| \frac{\left(P_{1}+2 Q_{2}\left(V_{g} q_{1}+\omega g \sigma(b)\right)\right.}{\left(g b-V_{g}^{2}\right)}+O\left(K^{4}\right) .
$$

The numerical search reveals that the growth rate of instability increases in regions $U_{1}$ and $R_{1}$ whereas it decreases in region $R_{2}$ when there is an increase either in the magnetic field strength or the permeability of the medium. The growth rate of the instability of a mode which is sinusoidal in $\eta$ and antisymmetric in $\xi$ gives rise to the bending of the wave crest, i.e., producing a "snake effect" or "sausage instability" about its undisturbed position. The crest of the disturbances oscillates to and fro in the $\xi-\eta$-plane about the equilibrium position. Whether this effect is enhanced or checked depends on the effect of the magnetic field in the unstable regions $U_{1}, R_{1}$, and $R_{2}$. In the instability bands $U_{2}$ and $U_{3}$, the group velocity rate $P$ is positive and the envelope soliton is unstable with respect to long symmetric even perturbations. The growth rate of instability with wave number $K$ and frequency $\Omega$ is furnished by

$$
\Omega^{2}=-2 K^{2} a^{2} P P_{1}+O\left(K^{4}\right) .
$$


Such a growth of this mode induces periodicity in modulation of the wave amplitude $a$ in $\eta$. In the region $U_{2}$, the growth rate increases with increase in $V_{A}^{2}$ for small depth limit, and decreases with $V_{A}^{2}$ for deep depth limit. In the region $U_{3}$, the growth rate decreases with increase in the magnetic field strength thus asserting a destabilizing influence.

The one-dimensional waveguide solutions are possible in the regions $U_{2}, S_{3}$, and $U_{3}$ because $P, P_{1}$ are both positive and $Q_{1}$ is negative. The effect of the magnetic field is similar to that in the case of envelope solitons but with the role of $\xi$ and $\eta$ interchanged. The instability described above may lead to a focussing phenomenon.

5. Nonlinear focussing. We shall now dwell on the nonlinear focussing which emerges out of the solutions of Eqs. (25) and (26) obtained in the previous section. Following Zakharov [14], we define the two integrals of motion

$$
\begin{gathered}
N=\iint|A|^{2} d \xi d \eta \\
H=\frac{1}{2} \iiint\left[P\left|\frac{\partial A}{\partial \xi}\right|^{2}+P_{1}\left|\frac{\partial A}{\partial \eta}\right|^{2}+\frac{Q_{1}}{4}|A|^{4}\right. \\
\left.+\left(\frac{\left(V_{g}^{2}-g b\right)}{\beta}\left|\frac{\partial \phi}{\partial \xi}\right|^{2}+\frac{1}{\beta}\left|\frac{\partial \phi}{\partial \eta}\right|^{2}\right) \cdot Q_{2}^{3}\right] d \xi d \eta, \\
\beta=\left(2 V_{g} q_{1}+\omega \sigma(b)\right),
\end{gathered}
$$

where $N$ is the wave action and $H$ is the Hamiltonian. Using (56) and (57) into (25) and (26), we deduce the virial equation

$$
\frac{\partial^{2} I}{\partial \tau^{2}}=4 H
$$

where the moment of inertia $I$ is defined as

$$
I=\iint\left(\xi^{2}+\eta^{2}\right)|A|^{2} d \xi d \eta
$$

Equation (58) upon integration yields

$$
\langle I\rangle=\frac{2 \tau^{2} H}{N}+C_{1} \tau+C_{2},
$$

where

$$
\langle I\rangle=\frac{I}{N} .
$$

The constants $C_{1}$ and $C_{2}$ in (60) are determined by the initial conditions

$$
C_{1}=\left.\frac{\partial}{\partial \tau}\langle I\rangle\right|_{\tau=0}, \quad C_{2}=\left.\langle I\rangle\right|_{\tau=0}=I(0) .
$$

Note that $C_{2}$ is always positive. The sufficient condition for the collapse to occur in finite time is that $\langle I\rangle$ tends to zero. That happens anywhere where $|A| \rightarrow 0$ except at the point of the collapse called the focus. The condition for the focussing or the emergence of singularity is the vanishing of the right-hand side of Eq. (58) 
at a certain time. This requirement can be satisfied by a wide class of initial data. When $H<0$, the quantity $Q<0$ in Eq. (57), leading to a singularity at a time, say $\tau=\tau_{0}^{*}$. The quantity $Q$ decreases further when the magnetic field is increased in the region $U_{2}$. In this region, it is easier to observe the self-focussing phenomenon for magnetic fluids as compared to ideal fluids. In other regions, no specific conclusions can be drawn, and the problem is still open.

6. Long-short wave interactions. We shall now investigate the nonlinear resonant interactions between the long and the short waves. Equation (25) has a singularity when the group velocity of the wave packet in the magnetic fluid coincides with the phase velocity of the long gravity waves. This resonant interaction results in the breakdown of the usual perturbation expansions taken in Sec. 2. To surmount this difficulty, we introduce the multiple scales

$$
\begin{array}{cc}
x_{1}=\varepsilon^{2 / 3} x_{0}, & x_{2}=\varepsilon^{4 / 3} x_{0}, \\
t_{1}=\varepsilon^{2 / 3} t_{0}, & t_{2}=\varepsilon^{4 / 3} t_{0} .
\end{array}
$$

The variables $\phi, \psi$, and $\eta$ are represented in terms of asymptotic series

$$
\begin{gathered}
\phi=\sum_{n=0}^{6} \varepsilon^{(n+2) / 3} \Phi_{n}+O\left(\varepsilon^{3}\right), \\
\psi=\sum_{n=1}^{6} \varepsilon^{(n+2) / 3} \psi_{n}+O\left(\varepsilon^{3}\right), \\
\eta=\sum_{n=1}^{6} \varepsilon^{(n+2) / 3} \eta_{n}+O\left(\varepsilon^{3}\right) .
\end{gathered}
$$

We will restrict ourselves to the one-dimensional case only. It is interesting to observe that by choosing the scales as above, the lowest order mode $\Phi_{0}$ and the next order mode $\Phi_{1}$ describe the long and the short wave free modes, respectively. The interactions between these two modes will occur at the higher order. Up to $O\left(\varepsilon^{2}\right)$, the long wave mode is not influenced by the presence of the short wave mode. Proceeding as in the previous section, we realize that the significant interactions occur at $O\left(\varepsilon^{7 / 3}\right)$, and the nonsecularity condition for the amplitude is

$$
i \frac{\partial A}{\partial \tau}+P \frac{\partial^{2} A}{\partial \xi^{2}}=\chi A \frac{\partial \Phi_{0}}{\partial \xi}
$$

where

$$
\begin{gathered}
\chi=-\frac{k}{2 \omega \sigma(b)}\left[\left(\omega^{2}\left(1-\sigma^{2}(b)\right)+\frac{k^{2} V_{A}^{2}}{2}\right.\right. \\
\left.\left.\times(\mu-1)\left\{2 B \sigma(b)+\frac{2\left(l_{11}-l_{22}\right) \sigma(b)}{(\mu \sigma(b)+1)}\right\}\right) V_{g} g^{-1}-2 \omega \sigma(b)\right], \\
\tau=t_{2}, \quad \xi=x_{1}-V_{g} t_{1} .
\end{gathered}
$$



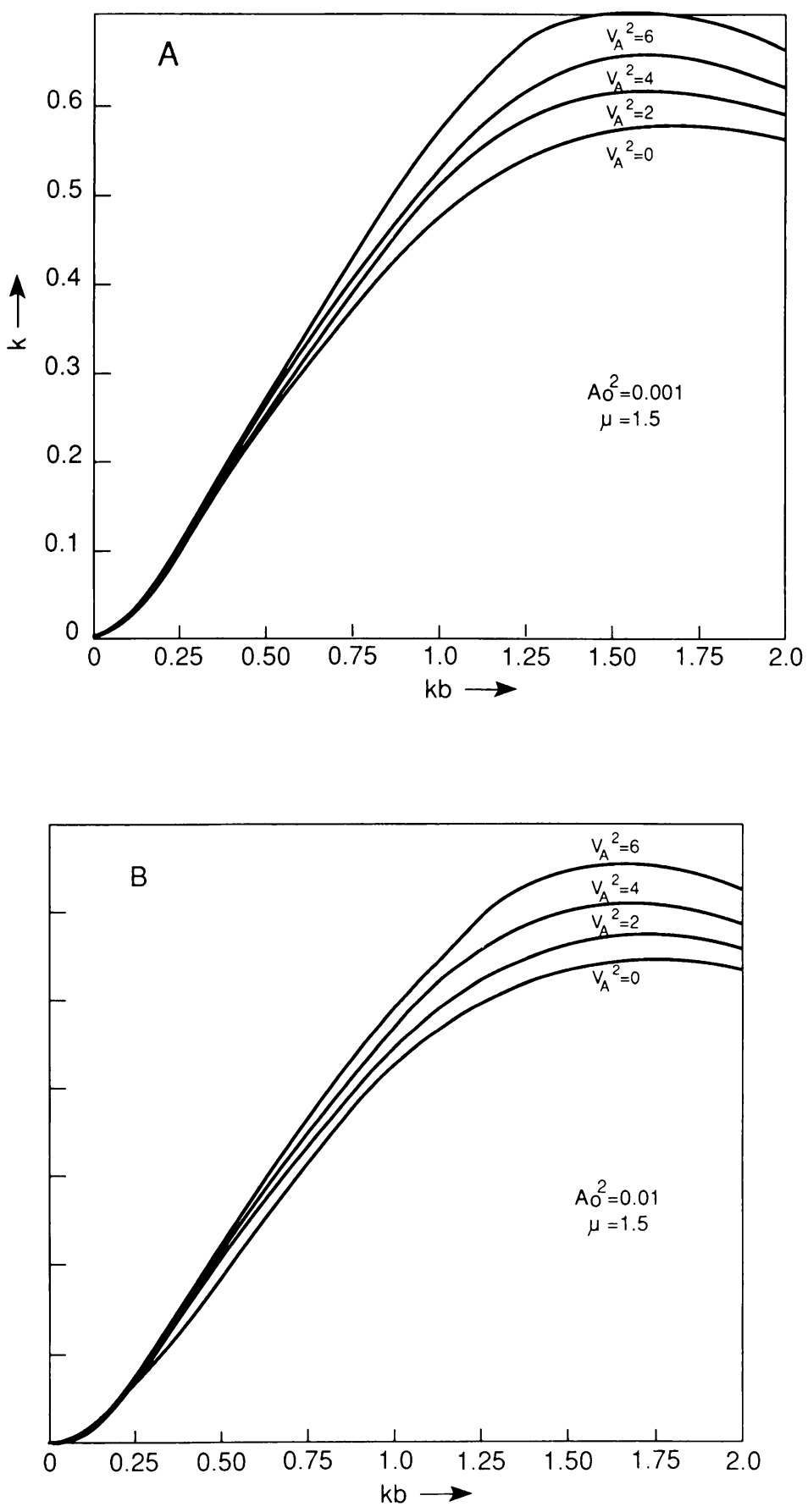

FIG. 3. The stability diagram for the long and the short wave resonance interactions for various values of $I_{4}^{2}$. The stability region is depicted below the curves. 
To find the evolution of the long wave mode, we proceed to $O\left(\varepsilon^{2 / 3}\right)$. From the kinematic condition (6), we obtain

$$
\frac{\partial^{2}}{\partial \tau \partial \xi}\left(\Phi_{0}\right)=-\frac{k^{2}}{2}\left(1-\frac{1}{\sigma^{2}}\right) \frac{\partial|A|^{2}}{\partial \xi} .
$$

The resonant interaction between the short and the long wave is governed by the two coupled equations (68) and (71). On setting $B=\chi\left(\partial \Phi_{0} / \partial \xi\right)$, we find

$$
i \frac{\partial A}{\partial \tau}+P \frac{\partial^{2} A}{\partial \xi^{2}}=B A,
$$

and

$$
\frac{\partial B}{\partial \tau}=-\alpha \frac{\partial}{\partial \xi}|A|^{2}
$$

where

$$
\alpha=\frac{1}{2} \chi k^{2}\left(1-\frac{1}{\sigma^{2}}\right) \text {. }
$$

The progressive wave train solutions of Eqs. (72) and (73) are unstable (see Benney [18] and Djordjevic and Redekopp [9]) when

$$
P^{2} k^{3}<3^{3 / 2}|\alpha|\left|A_{0}\right|^{2}
$$

where $A_{0}$ is the initial value of the amplitude. The inequality (75) is still valid even if the long wave amplitude is initially absent, implying thereby that the short waves of finite amplitude interact among themselves to produce a long wave mode.

In Fig. 3, we have sketched the transition curve for $S_{2}=P^{2} K^{3}-(3)^{3 / 2}|\alpha|\left|A_{0}\right|^{2}=0$ with $\left|A_{0}\right|^{2}=0.01$ and $\left|A_{0}\right|^{2}=0.001$ in the neighbourhood of the wavenumbers satisfying the resonance condition. Compared to ideal fluids, the presence of the magnetic field decreases the region of instability, thereby exerting the stabilizing influence. As remarked earlier, this phenomenon is absent in the deep magnetic fluid limit.

The set of Eqs. (72) and (73) can be integrated in terms of the Jacobian elliptic function. The phase jump and the solitary wave can then be derived as special cases. Ma [24] has demonstrated that for ideal fluids, these equations can be solved by using the inverse scattering technique resulting in soliton solution. For the magnetic fluid case, the coefficients appearing in Eqs. (72), (73) are dependent upon the magnetic permeability and the applied magnetic field strength. Like ideal fluids, an arbitrary initial disturbance for magnetic fluids will also lead to a series of solitons. By increasing the strength of the magnetic field, the generation of solitons can be suppressed.

7. Conclusions. We have shown that the solitons and "waveguides" which exist in one space propagation are unstable to transverse perturbation in a magnetic fluid. When compared with ideal fluids, the growth rate of instability is enhanced in some regions and suppressed in others with an increase in the applied magnetic field strength or by using the magnetic fluid of higher permeability. Such an instability may lead to a self-focussing phenomenon, and can be easily observed in magnetic fluids. In ideal fluid experiments this kind of instability has not been seen so far. 
On the other hand, if $(k b)^{2} \ll \varepsilon$, i.e., long wave approximation, an equation similar to that of Kadomstev and Petviashvibi [25] for magnetic fluids can be derived. In the corner of the region $U_{1}$, in Fig. 1, we obtain $\mathrm{KdV}$ solitons in one space dimension [27-30]. Such a solution shall be unstable to transverse perturbations. The "lump" solutions are predicted in the region $S_{3}$ [26].

In the last section, we have revealed that, like ideal fluids, the long-short wave resonance phenomenon exists in magnetic fluids also, and gives rise to a significant energy exchange between a short wave mode and a long wave mode. The linearized analysis exhibits an instability, provided inequality (75) is satisfied for wavenumbers in the vicinity of the resonance. The terminal stage of this instability predicts the generation of solitons. The presence of the magnetic field inhibits the soliton production in the regions where they happen to exist.

\section{REFERENCES}

[1] R. E. Zelazo and J. R. Melcher, Dynamics and stability of ferrofluids: surface interactions, J. Fluid Mech. 39, 1 (1969)

[2] R. E. Rosensweig, Ferrohydrodynamics, Cambridge Univ. Press, New York, 1985

[3] R. E. Rosensweig, Magnetic fluids, Annal. Rev. Fluid Mech. 19. 437 (1987)

[4] R. E. Rosensweig, Fluid dynamics and science of magnetic liquids. Adv. Electronics and Electron Physics, 48, 103 (1979)

[5] S. K. Malik and M. Singh, Modulational instability in magnetic fluids, Quart. Appl. Math. 42, 57 (1985)

[6] S. K. Malik and M. Singh. Nonlinear capillary-gravity waves in magnetic fluids, Quart. Appl. Math. 47, 59-70 (1989)

[7] M. C. Shen and S. M. Sun, Ray method for surface waves on a ferromagnetic fluid, Wave Motion 9, 99 (1987)

[8] A. Davey and K. Stewartson. On three-dimensional packets of surface waves, Proc. Roy. Soc. London Ser. A 338, 101-110 (1974)

[9] V. D. Djordjevic and L. G. Redekopp. On two-dimensional packets of capillary-gravity waves, J. Fluid Mech. 79, 703 (1977)

[10] M. J. Ablowitz and H. Segur, On the evolution of packets of water waves. J. Fluid Mech. 92.691 (1979)

[11] M. J. Ablowitz and H. Segur, Solitons and the Inverse Spectral Transform. SIAM, 1981

[12] S. V. Manakov, Dynamics of classical solitons, Phys. Rep. 35, 1 (1978)

[13] V. E. Zakharov and S. Synakh, The nature of the selffocussing singularity, Soviet Phys. JETP 41. $465(1975)$

[14] V. E. Zakharov, Collapse of Langmuir waves, Soviet Phys. JETP 72. 908 (1972)

[15] F. H. Berkshire and J. D. Gibbon. Collapse in the n-dimensional nonlinear Schrödinger equation. Stud. Appl. Math. 69, 229 (1983)

[16] S. K. Malik and M. Singh, Nonlinear focussing in magnetic fluids, Quart. Appl. Math. 44, 629-637 (1987)

[17] D. J. Benney, Significant interaction hetween small and large scale surface waves. Stud. Appl. Math. 55, 93-106 (1976)

[18] D. J. Benney, A general theory of interactions between short and long waves. Stud. Appl. Math. 56. $81-94(1977)$

[19] T. B. Benjamin and J. E. Feir, The disintegration of wave trains in decp water. J. Fluid Mech. 27. 417 (1967)

[20] A. C. Newell, Longwaves-shortwaves, a solvable model. SIAM J. Appl. Math. 35, 650 (1978)

[21] R. Kant and S. K. Malik, Nonlinear internal resonance in magnetic fluids. J. Mag. Mat. 65, 347 (1987)

[22] V. E. Zakharov and A. B. Shabat, Exact theory of two-dimensional self-focussing and one-dimensinal self-modulating waves in nonlinear media. Soviet Phys. JETP 34. 62 (1972) 
[23] V. E. Zakharov and A. M. Rubenchik, Instability of waveguides and solutions in nonlinear media, Soviet Phys. JETP 38, 494 (1974)

[24] Y. C. Ma, The complete solution of the long wave-short wave resonance equations, Stud. Appl. Math. 59, 201 (1978)

[25] B. B. Kadomstev and V. I. Petviashvibi, On the stability of solitary waves in weakly dispersive media, Soviet Phys. Dokl. 15, 539 (1970)

[26] S. K. Malik and M. Singh, On long surface waves in magnetic fluids, to appear

[27] J. Hammack and H. Segur, The Korteweg-de Vries equation and water waves, J. Fluid Mech. 65, 289 (1974)

[28] J. Hammack and H. Segur, The Korteweg-de Vries equation and water waves. Part 3, Oscillatory Waves, J. Fluid Mech. 84, 337 (1978)

[29] H. Segur, in Topics in Ocean Physics (A. R. Osborne, ed.), North Holland, New York, 1982

[30] M. C. Shen, Solitons on a magnetic fluid, Continuum Mechanics and Its Application (G. A. C. Graham and S. K. Malik, eds.), Hemisphere, New York, 1989 